\title{
Conformal Metrics with Constant $Q$-Curvature
}

Andrea MALCHIODI

SISSA, Via Beirut 2-4, Trieste, Italy

E-mail: malchiod@sissa.it

URL: http://people.sissa.it/ malchiod/

Received September 02, 2007, in final form December 05, 2007; Published online December 13, 2007

Original article is available at http://www.emis.de/journals/SIGMA/2007/120/

\begin{abstract}
We consider the problem of varying conformally the metric of a four dimensional manifold in order to obtain constant $Q$-curvature. The problem is variational, and solutions are in general found as critical points of saddle type. We show how the problem leads naturally to consider the set of formal barycenters of the manifold.
\end{abstract}

Key words: $Q$-curvature; geometric PDEs; variational methods; min-max schemes

2000 Mathematics Subject Classification: 35B33; 35J35; 53A30; 53C21

Dedicated to Tom Branson

\section{Introduction}

$Q$-curvature is a scalar quantity introduced by Tom Branson in 1985, [5], which in recent years has attracted lot of attention since it turns out to be fundamental in several questions. Here we will be mainly interested in its role in conformal geometry, and especially in its transformation law under a conformal change of metric.

Let $(M, g)$ be a four dimensional manifold, with Ricci tensor $\operatorname{Ric}_{g}$, scalar curvature $R_{g}$ and Laplace-Beltrami operator $\Delta_{g}$ : the $Q$-curvature of $M$ is defined by

$$
Q_{g}=-\frac{1}{12}\left(\Delta_{g} R_{g}-R_{g}^{2}+3\left|\operatorname{Ric}_{g}\right|^{2}\right)
$$

If we perform the conformal change of metric $\tilde{g}=e^{2 w} g$, for some smooth function $w$ on $M$, then the $Q$-curvature transforms in the following way

$$
P_{g} w+2 Q_{g}=2 Q_{\tilde{g}} e^{4 w},
$$

where $P_{g}$ is the Paneitz operator

$$
P_{g}(\varphi)=\Delta_{g}^{2} \varphi+\operatorname{div}_{g}\left(\frac{2}{3} R_{g} g-2 \operatorname{Ric}_{g}\right) d \varphi, \quad \varphi \in C^{\infty}(M)
$$

introduced in [43]. When we modify conformally $g$ as before, also $P_{g}$ obeys a simple transformation law, which is the following

$$
P_{\tilde{g}}=e^{-4 w} P_{g} .
$$

The last formula and (1) are indeed analogous to classical ones which hold on compact surfaces: in fact, if we let $K_{g}$ denote the Gauss curvature of a surface $\Sigma$ it is well known that

$$
\Delta_{\tilde{g}}=e^{-2 w} \Delta_{g}, \quad-\Delta_{g} w+K_{g}=K_{\tilde{g}} e^{2 w} .
$$

${ }^{\star}$ This paper is a contribution to the Proceedings of the 2007 Midwest Geometry Conference in honor of Thomas P. Branson. The full collection is available at http://www.emis.de/journals/SIGMA/MGC2007.html 
In addition to these, we have an analogy with the classical Gauss-Bonnet formula $(\chi(\Sigma)$ stands here for the Euler characteristic of $\Sigma$ )

$$
\int_{\Sigma} K_{g} d V_{g}=2 \pi \chi(\Sigma)
$$

Indeed, if $W_{g}$ denotes the Weyl tensor of a four manifold $(M, g)$, one has

$$
\int_{M}\left(Q_{g}+\frac{\left|W_{g}\right|^{2}}{8}\right) d V_{g}=4 \pi^{2} \chi(M)
$$

Notice that, from either (1) or (2), it follows that the total $Q$-curvature of $M$, which we denote by

$$
k_{P}=\int_{M} Q_{g} d V_{g}
$$

is a conformal invariant of $(M, g)$ : we refer for example to the survey [19] for more details.

Since for two-dimensional surfaces the integral of the Gauss curvature characterizes completely the topology, it is reasonable to expect that conformal invariant objects on four manifolds, like $P_{g}$ or $k_{P}$, could at least provide some partial geometric information. Indeed some results in this spirit were obtained by Gursky in [34] (see also [33]). If a manifold of non-negative Yamabe class $Y(g)$ satisfies also $k_{P} \geq 0$, then ker $P_{g}$ consists only of the constant functions and $P_{g} \geq 0$, namely $P_{g}$ is a non-negative operator. If in addition $Y(g)>0$, then the first Betti number of $M$ vanishes, unless $(M, g)$ is conformally equivalent to a quotient of $S^{3} \times \mathbb{R}$. On the other hand, if $Y(g) \geq 0$, then $k_{P} \leq 8 \pi^{2}$ with equality holding if and only if $(M, g)$ is conformally equivalent to the standard sphere.

In two dimensions, the uniformization theorem asserts that every compact surface carries a conformal metric with constant Gauss curvature. Such deformation has a strong geometric relevance, since the deformed metric will be a model one: spherical, Euclidean or hyperbolic. In four dimensions one might ask the same question for the $Q$-curvature: this being a scalar quantity, it will not be sufficient to control the whole curvature tensor, however the new metric will be a privileged one and might have special properties.

Writing $\tilde{g}=e^{2 w} g$, by (1) the question amounts to finding a solution of the equation

$$
P_{g} w+2 Q_{g}=2 \bar{Q} e^{4 w},
$$

where $\bar{Q}$ is a real constant. Problem (3) is variational, and solutions can be found as critical points of the following functional

$$
I I(u)=\left\langle P_{g} u, u\right\rangle+4 \int_{M} Q_{g} u d V_{g}-k_{P} \log \int_{M} e^{4 u} d V_{g}, \quad u \in H^{2}(M) .
$$

Here $H^{2}(M)$ is the space of real functions on $M$ which are of class $L^{2}$ together with their first and second derivatives, and the symbol $\left\langle P_{g} u, v\right\rangle$ stands for

$$
\left\langle P_{g} u, v\right\rangle=\int_{M}\left(\Delta_{g} u \Delta_{g} v+\frac{2}{3} R_{g} \nabla_{g} u \cdot \nabla_{g} v-2\left(\operatorname{Ric}_{g} \nabla_{g} u, \nabla_{g} v\right)\right) d V_{g}, \quad u, v \in H^{2}(M) .
$$

Notice that the functional $I I$ is well defined on $H^{2}(M)$ : in fact, by the Adams inequality (see $[1,18])$ one has

$$
\log \int_{M} e^{4(u-\bar{u})} d V_{g} \leq \frac{1}{8 \pi^{2}}\left\langle P_{g} u, u\right\rangle+C, \quad P_{g} \geq 0, \quad u \in H^{2}(M),
$$


where $\bar{u}$ is the average of $u$ on $M$ and where $C$ depends only on $M$, so the last term in $I I$ is well defined in this Sobolev space.

Problem (3) has been solved in [18] for the case in which $P_{g}$ is a non-negative operator and when $k_{P}<8 \pi^{2}$ : by the above-mentioned result of Gursky, sufficient conditions for these assumptions to hold are that $Y(g) \geq 0$ and that $k_{P} \geq 0$ (and $(M, g)$ is not conformal to the standard sphere). More general sufficient conditions for the above hypotheses to hold have been obtained by Gursky and Viaclovsky in [35].

Under the assumptions in [18], by (4) the functional $I I$ is bounded from below and coercive, hence solutions can be found as global minima. The result in [18] has also been extended in [10] to higher-dimensional manifolds (regarding higher-order operators and curvatures) using a geometric flow.

The solvability of (3), under the above hypotheses, is useful in the study of some conformally invariant fully non-linear equations, as shown in [13]. Some remarkable geometric consequences of this study, given in [12, 13], are the following. If a manifold of positive Yamabe class satisfies $k_{P}>0$, then there exists a conformal metric with positive Ricci tensor, and hence $M$ has finite fundamental group. Furthermore, under the additional quantitative assumption $k_{P}>\frac{1}{8} \int_{M}\left|W_{g}\right|^{2} d V_{g}, M$ must be diffeomorphic to the standard four-sphere or to the standard projective space.

As we already mentioned, $Q$-curvature (with its higher-dimensional versions and the Paneitz operator) is relevant in the study of many other questions, like log-determinant formulas, compactification of locally conformally flat manifolds, Poincaré-Einstein metrics, ambient metrics, tractor calculus, volume renormalization, scattering theory and others, see the (incomplete) list of references [4, 6, 7, 8, 16, 17, 26, 27, 28, 29, 30, 31, 32].

This note concerns some recent progress about problem (3), in particular an extension of the uniformization result of [18], which is given in [24].

Theorem 1. Suppose ker $P_{g}=\{$ const $\}$, and assume that $k_{P} \neq 8 k \pi^{2}$ for $k=1,2, \ldots$ Then $(M, g)$ admits a conformal metric with constant $Q$-curvature.

The assumptions in Theorem 1 are conformally invariant and generic, and hence apply to a large class of four manifolds, especially to some with negative curvature. For example, products of two negatively-curved surfaces might have total $Q$-curvature greater than $8 \pi^{2}$, see [23]. However the theorem does not cover all manifolds, for example conformally flat manifolds with positive and even Euler characteristic, by (2), are excluded.

Our assumptions include those made in [18] and one (or both) of the following two possibilities

$$
\begin{aligned}
& k_{P} \in\left(8 k \pi^{2}, 8(k+1) \pi^{2}\right) \quad \text { for some } k \in \mathbb{N} ; \\
& P_{g} \text { possesses } \bar{k} \text { (counted with multiplicity) negative eigenvalues. }
\end{aligned}
$$

In these cases the functional $I I$ is unbounded from below, and hence it is necessary to find critical points which are possibly of saddle type. This is done using a new min-max scheme, which we are going to describe briefly in the next section, depending on $k_{P}$ and the spectrum of $P_{g}$ (in particular on the number of negative eigenvalues $\bar{k}$, counted with multiplicity).

One fundamental issue in applying variational techniques is compactness: it is possible to prove that indeed solutions of (3) (or of some perturbation) stay compact provided $P_{g}$ has no kernel and $k_{P}$ stay bounded away from $8 \pi^{2} \mathbb{N}$. The following result was proved in $[25,37]$ using blow-up analysis.

Theorem 2 ([37]). Suppose $\operatorname{ker} P_{g}=\{$ const $\}$ and that $\left(u_{l}\right)_{l}$ is a sequence of solutions to

$$
P_{g} u_{l}+2 Q_{l}=2 k_{l} e^{4 u_{l}} \quad \text { in } M,
$$


satisfying $\int_{M} e^{4 u_{l}} d V_{g}=1$, where $k_{l}=\int_{M} Q_{l} d V_{g}$, and where $Q_{l} \rightarrow Q_{0}$ in $C^{0}(M)$. Assume also that

$$
k_{0}:=\int_{M} Q_{0} d V_{g} \neq 8 k \pi^{2} \quad \text { for } k=1,2, \ldots
$$

Then $\left(u_{l}\right)_{l}$ is bounded in $C^{\alpha}(M)$ for any $\alpha \in(0,1)$.

We will show below how this result is useful in deriving existence. For reasons of brevity, we will describe the proof of Theorem 1 only: as mentioned, we will find in general solutions as saddle points of $I I$. To use variational techniques we will try to understand the topological properties of the sublevels, in particular the very negative ones.

If $k_{P}>8 \pi^{2}$, it is easy to see that $I I$ is unbounded from below: one can fix a point $x \in M$ and consider a test function with the following form

$$
\varphi_{\lambda, x}(y) \simeq \frac{1}{4} \log \left(\frac{2 \lambda}{1+\lambda^{2} \operatorname{dist}(y, x)^{2}}\right), \quad y \in M,
$$

where $\operatorname{dist}(\cdot, \cdot)$ denotes the metric distance on $M$. For $\lambda \rightarrow+\infty$ we have that $I I\left(\varphi_{\lambda, x}\right) \rightarrow-\infty$. Another way to attain large negative value of $I I$ is to consider negative eigenvectors of $P_{g}$ with large norm: in the next section we will focus on the first alternative only, and reason looking at how the function $e^{4 u}$ is distributed over different regions of $M$.

The last section describes other related results and some open problems in this direction.

\section{Sketch of the proof}

We give here the main ideas for the proof of Theorem 1: throughout this section we assume for simplicity that $P_{g}$ is positive definite (except on constants), referring to [24] for details when negative eigenvalues are present.

\subsection{Improved Adams inequality and applications}

One of the main ingredients in our proof is an improvement of the Adams inequality on the functions $u$ for which $e^{4 u}$ is spread into multiple regions: here is the precise statement.

Lemma 1. For a fixed integer $\ell$, let $\Omega_{1}, \ldots, \Omega_{\ell+1}$ be subsets of $M$ satisfying $\operatorname{dist}\left(\Omega_{i}, \Omega_{j}\right) \geq \delta_{0}$ for $i \neq j$, where $\delta_{0}$ is a positive real number, and let $\gamma_{0} \in\left(0, \frac{1}{\ell+1}\right)$. Then, for any $\tilde{\varepsilon}>0$ there exists a constant $C=C\left(\ell, \tilde{\varepsilon}, \delta_{0}, \gamma_{0}\right)$ such that

$$
\log \int_{M} e^{4(u-\bar{u})} d V_{g} \leq C+\frac{1}{8(\ell+1) \pi^{2}-\tilde{\varepsilon}}\left\langle P_{g} u, u\right\rangle
$$

for all the functions $u \in H^{2}(M)$ satisfying

$$
\frac{\int_{\Omega_{i}} e^{4 u} d V_{g}}{\int_{M} e^{4 u} d V_{g}} \geq \gamma_{0}, \quad \forall i \in\{1, \ldots, \ell+1\}
$$

The original improvement argument is given in [21], where the authors treat $H^{1}$ functions on surfaces and the case $\ell=1$ : some modifications are needed here to deal with the fourth order operator. The main step of the proof consists in constructing cutoff functions $g_{i}$ which are identically equal to 1 on $\Omega_{i}$ and which have mutually disjoint supports. Then one applies (4) to $g_{i} u$ and chooses the index $i$ so that $\int_{M} g_{i}^{2}(\Delta u)^{2} d V_{g}$ is minimal: the remaining terms of $\int_{M}\left(\Delta\left(g_{i} u\right)\right)^{2} d V_{g}$ can be treated as perturbations and yield $o\left(\|u\|_{H^{2}(M)}\right)$. 


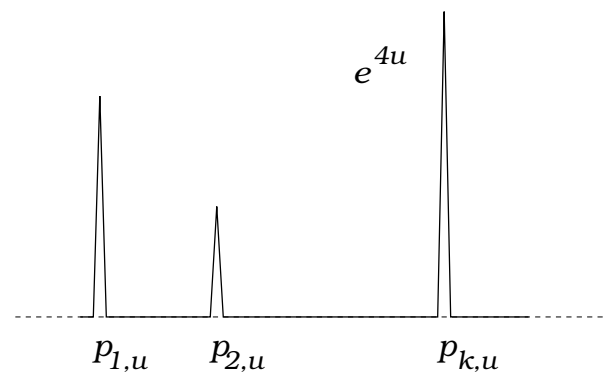

Figure 1. Shape of functions with low energy.

The above lemma states that if $k_{P}<8(k+1) \pi^{2}$ for some natural $k$, and if $u$ satisfies (7) with $\ell=k$, then $I I(u)$ stays bounded from below. Since we can choose $\delta_{0}$ and the $\Omega_{i}$ 's arbitrarily, this suggests that if $I I(u)$ is sufficiently low, then $e^{4 u}$ has to concentrate near at most $k$ points of $M$. Using a covering argument this can indeed be made rigorous, yielding the following result.

Lemma 2. Assuming $P_{g} \geq 0$ and $k_{P} \in\left(8 k \pi^{2}, 8(k+1) \pi^{2}\right)$ with $k \geq 1$, the following property holds. For any $\varepsilon>0$ and any $r>0$ there exists a large positive $L=L(\varepsilon, r)$ such that for every $u \in H^{2}(M)$ with $I I(u) \leq-L$ there exist $k$ points $p_{1, u}, \ldots, p_{k, u} \in M$ such that

$$
\int_{M \backslash \cup_{i=1}^{k} B_{r}\left(p_{i, u}\right)} e^{4 u} d V_{g}<\varepsilon .
$$

Lemma 2 basically states that if $I I(u)$ is large negative, then the conformal volume $e^{4 u}$ has a shape as in Fig. 1.

If we assume the normalization $\int_{M} e^{4 u} d V_{g}=1$, the figure suggests to consider the following set

$$
M_{k}=\left\{\sum_{i=1}^{k} t_{i} \delta_{x_{i}}: x_{i} \in M, t_{i} \in[0,1], \sum_{i=1}^{k} t_{i}=1\right\},
$$

known in literature as the set of formal barycenters of $M$. For $k=1 M_{1}$ is homeomorphic to $M$, but for larger $k$ 's this is a stratified set, namely union of open manifolds of different dimensions, whose maximal one is $5 k-1$. For further properties of these sets we refer the reader to [3, 9]: we are going to need especially the following one, which can be proved with an argument in algebraic topology.

Lemma 3 (well-known). If $M$ is a compact manifold, for any $k \geq 1$ the set $M_{k}$ is noncontractible.

Using Lemma 2 one can construct a continuous (and non-trivial) map from very negative sublevels of $I I$ into $M_{k}$.

Proposition 1. For $k \geq 1$ (see (5)) there exists a large $L>0$ and a continuous map $\Psi$ from the sublevel $\{I I \leq-L\}$ into $M_{k}$ which is topologically non-trivial.

By topologically non-trivial we mean that the action of $\Psi_{*}$ on the homology classes of the sublevel $\{I I \leq-L\}$ has non-trivial image. The proof of the latter proposition is rather involved and takes some long technical work: we limit ourselves to explain some of the ideas for the construction. It is easy to project when $e^{4 u}$ is close to smooth pieces of $M_{k}$, but some difficulties may occur when approaching the singularities. In this case, one can project first on the subsets of lowest possible dimension, and then take homotopies with the higher-dimensional ones, see Fig. 2. 


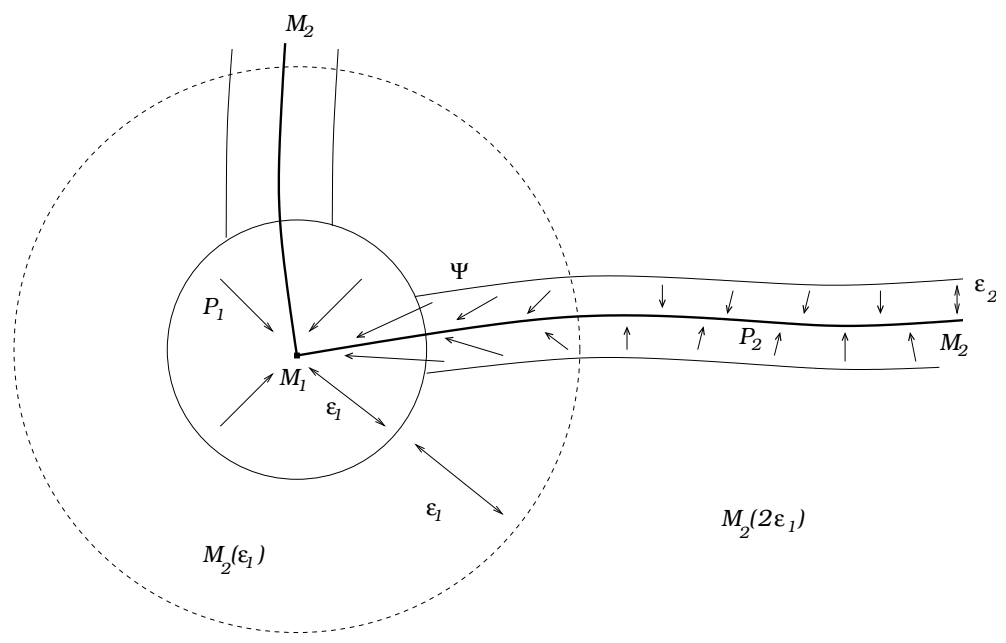

Figure 2. A sketch on the construction of $\Psi$ for $k=2$.

\subsection{The min-max scheme}

In order to proceed we need some preliminary notation. For $\delta>0$ small, consider a smooth non-decreasing cut-off function $\chi_{\delta}: \mathbb{R}_{+} \rightarrow \mathbb{R}$ satisfying the following properties

$$
\begin{aligned}
& \chi_{\delta}(t)=t \quad \text { for } t \in[0, \delta] ; \\
& \chi_{\delta}(t)=2 \delta \quad \text { for } t \geq 2 \delta ; \\
& \chi_{\delta}(t) \in[\delta, 2 \delta] \quad \text { for } t \in[\delta, 2 \delta] .
\end{aligned}
$$

Then, given $\sigma \in M_{k}\left(\sigma=\sum_{i=1}^{k} t_{i} \delta_{x_{i}}\right)$ and $\lambda>0$, we define the function $\varphi_{\lambda, \sigma}: M \rightarrow \mathbb{R}$ as

$$
\varphi_{\lambda, \sigma}(y)=\frac{1}{4} \log \sum_{i=1}^{k} t_{i}\left(\frac{2 \lambda}{1+\lambda^{2} \chi_{\delta}^{2}\left(d_{i}(y)\right)}\right)^{4}, \quad y \in M,
$$

where $d_{i}(y)=\operatorname{dist}\left(y, x_{i}\right), y \in M$, with $\operatorname{dist}(\cdot, \cdot)$ denoting the distance function on $M$. We have then the following result.

Proposition 2. Let $\varphi_{\lambda, \sigma}$ be defined as in (8). Then, as $\lambda \rightarrow+\infty$ the following properties hold

(i) $e^{4 \varphi_{\lambda, \sigma}} \rightarrow \sigma$ weakly in the sense of distributions;

(ii) $I I\left(\varphi_{\lambda, \sigma}\right) \rightarrow-\infty$ uniformly for $\sigma \in M_{k}$.

Moreover, if $\Psi$ is given in Proposition 1, the map $\sigma \mapsto \varphi_{\lambda, \sigma} \mapsto \Psi\left(\varphi_{\lambda, \sigma}\right)$ converges to the identity on $M_{k}$ as $\lambda$ tends to infinity.

It is indeed the last property, together with the non-contractibility of $M_{k}$, which guarantees the non-triviality of the map $\Psi$.

We next introduce the variational scheme which provides existence of solutions for (3). Let $\widehat{M}_{k}$ denote the (contractible) topological cone over $M_{k}$, which can be represented as $\widehat{M}_{k}=M_{k} \times[0,1]$ with $M_{k} \times\{0\}$ collapsed to a single point. Let first $L$ be so large that Proposition 1 applies with $\frac{L}{4}$, and then let $\bar{\lambda}$ be so large that Proposition 2 applies for this value of $L$. Fixing this number $\bar{\lambda}$, we define the following class of maps

$$
\Pi_{\bar{\lambda}}=\left\{\pi: \widehat{M}_{k} \rightarrow H^{2}(M): \pi \text { is continuous and } \pi(\cdot \times\{1\})=\varphi_{\bar{\lambda},} \text {, on } M_{k}\right\} .
$$

We have then the following properties. 
Lemma 4. The set $\Pi_{\bar{\lambda}}$ is non-empty and moreover, letting

$$
\bar{\Pi}_{\bar{\lambda}}=\inf _{\pi \in \Pi_{\bar{\lambda}}} \sup _{m \in \bar{M}_{k}} I I(\pi(m)), \quad \text { one has } \quad \bar{\Pi}_{\bar{\lambda}}>-\frac{L}{2} .
$$

As a consequence, the functional II possesses a Palais-Smale sequence at level $\bar{\Pi}_{\bar{\lambda}}$.

To check that $\Pi_{\bar{\lambda}}$ is non empty, it is sufficient to consider the map $\pi(t, \sigma)=t \varphi_{\bar{\lambda}, \sigma}$, which is well defined on $\widehat{M}_{k}$. A Palais-Smale sequence at level $c$ is a sequence $\left(u_{n}\right)_{n}$ such that $I I\left(u_{n}\right) \rightarrow c$ and such that $I I^{\prime}\left(u_{n}\right) \rightarrow 0$ as $n \rightarrow+\infty$. To produce it one considers any map in $\Pi_{\bar{\lambda}}$ with $\sup _{m \in \widehat{M_{k}}} I I(\pi(m))$ sufficiently close to $\bar{\Pi}_{\bar{\lambda}}$ and let it evolve via the gradient flow of $I I$, keeping its boundary fixed. By (9) the maximum value of $I I$ on the evolved set, which still belongs to $\Pi_{\bar{\lambda}}$, cannot go below $\bar{\Pi}_{\bar{\lambda}}$, so the gradient of $I I$ along the evolution has to become small somewhere along the flow, see for example [45].

If Palais-Smale sequences are bounded, then it is easy to see that they have to converge to a solution of (3). Unfortunately boundedness is not known in general, so we tackle this problem using an argument due to M. Struwe, see [44]. For $\rho$ in a neighborhood of 1 we define the functional $I I_{\rho}: H^{2}(M) \rightarrow \mathbb{R}$ by

$$
I I_{\rho}(u)=\left\langle P_{g} u, u\right\rangle+4 \rho \int_{M} Q_{g} d V_{g}-4 \rho k_{P} \log \int_{M} e^{4 u} d V_{g}, \quad u \in H^{2}(M),
$$

whose critical points give rise to solutions of the equation

$$
P_{g} u+2 \rho Q_{g}=2 \rho k_{P} e^{4 u} \quad \text { in } M .
$$

Running the above variational scheme one finds a min-max value as in Lemma 4, which we call $\bar{\Pi}_{\rho}$, and a corresponding Palais-Smale sequence. One can easily show that $\frac{\bar{\Pi}_{\rho}}{\rho}$ is nonincreasing, and hence differentiable, in $\left[1-\rho_{0}, 1+\rho_{0}\right]$ provided $\rho_{0}$ is sufficiently small. Using the following result, which can be proved as in [22], by the above comments and Theorem 2 one deduces then existence of solutions to (3).

Lemma 5. Let $\Lambda \subset\left[1-\rho_{0}, 1+\rho_{0}\right]$ be the (dense) set of $\rho$ for which the function $\frac{\bar{\Pi}_{\rho}}{\rho}$ is differentiable. Then for $\rho \in \Lambda I I_{\rho}$ possesses a bounded Palais-Smale sequence $\left(u_{l}\right)_{l}$ at level $\bar{\Pi}_{\rho}$.

A particular case of the above variational method has been used in [22] to study a mean field equations on compact surfaces, which in our setting would correspond to the case $k=1$.

\section{Final remarks}

The set of barycenters of a manifold (or of a set) has been used crucially in literature for the study of problems with lack of compactness, see [2, 3]. In particular, for Yamabe-type equations (including the Yamabe equation and several other applications), it has been employed to understand the structure of the critical points at infinity (or asymptotes) of the Euler functional, namely the way compactness is lost through a pseudo-gradient flow. Our use of the set $M_{k}$, although the map $\Psi$ presents some analogies with the Yamabe case, is of different type since it is employed to reach low energy levels and not to study critical points at infinity. As mentioned above, we consider a projection onto the $k$-barycenters $M_{k}$, but starting only from functions in $\{I I \leq-L\}$, whose concentration behavior is not as clear as that of the asymptotes for the Yamabe equation.

Theorem 1 has been recently extended to all dimensions in [40]: one of the issues there is that the explicit expression of the Paneitz operator is not known in general, but only the principal 
part is needed. Also, the blow-up analysis to obtain the counterpart of Theorem 2 relies almost entirely on the Green's representation formula.

The above approach has been also used in [41, 42] to study the case of four manifolds with boundary: in fact, in two papers by A. Chang and J. Qing, see [14, 15], it was constructed a scalar quantity, the T-curvature, which represents an analogue of the geodesic curvature for surfaces with boundary. If one wants to prescribe constant $Q$-curvature in the interior and zero $T$-curvature (and mean curvature) on the boundary, it turns out that the quantization of conformal volume at the boundary is one half of the one in the interior. As a consequence, one needs to modify the topological argument and consider formal barycenters which allow a double number of Dirac deltas on $\partial M$ compared to the interior.

We also mention the papers $[11,39,46]$, where the problem of prescribing non-constant $Q$-curvature on spheres was considered. In this case the analysis is more delicate since the problem is non compact, due to the presence of the Möbius group. One has then to analyze how compactness is lost and employ topological arguments, like degree of Morse theory, to derive existence results. In fact Morse theory can be used, jointly with the Poincaré-Hopf theorem, to compute the Leray-Schauder degree of (3). By Theorem 2, if $P_{g}$ has no kernel and if $k_{P} \notin 8 \pi^{2} \mathbb{N}$, then the solutions of (3) stay uniformly bounded, so the degree of the equation is well defined, and in [38] it was proven that it is given by

$$
\begin{aligned}
& (-1)^{\bar{k}} \quad \text { for } k_{P}<8 \pi^{2} ; \\
& (-1)^{\bar{k}}(-\chi(M)+1) \cdots(-\chi(M)+k) / k ! \quad \text { for } k_{P} \in\left(8 k \pi^{2}, 8(k+1) \pi^{2}\right), \quad k \in \mathbb{N},
\end{aligned}
$$

where $\bar{k}$ stands for the number of negative eigenvalues of $P_{g}$, counted with multiplicity. This result is related to one obtained in [20] for a mean field equation on compact surfaces, and based on sophisticated blow-up techniques: our proof is of different type and relies instead on Morse theory, as mentioned.

A natural question one may ask is whether Theorem 1 can be extended to the situations in which $P_{g}$ has non-trivial kernel or when $k_{P}$ is an integer multiple of $8 \pi^{2}$ : in this cases more precise characterizations of $P_{g}$ and $Q_{g}$ (or on their mutual relation) should be given.

One simple situation to consider is when $P_{g}$ has non-trivial kernel and $k_{P}=0$. In this case (3) has the following simple expression

$$
P_{g} u+2 Q_{g}=0 .
$$

Clearly, by Fredholm's alternative, the equation is solvable if and only if $Q_{g}$ is perpendicular to ker $P_{g}$. It seems possible to violate this condition but no example so far is known.

If one wishes to study the case of non-trivial kernel in more generality, possibly employing min-max arguments, it would be necessary to understand the behavior of $I I$ on $\operatorname{ker} P_{g}$, in particular on functions with large norm. For example, taking $v \in \operatorname{ker} P_{g}, v \neq 0$, one has

$$
I I(t v)=4 t \int_{M} Q v d V_{g}-k_{P} \log \int_{M} e^{4 t v} d V_{g}
$$

Since every element in ker $P_{g}$ is smooth we have $v \in L^{\infty}(M)$, and in the limit $t \rightarrow+\infty$ one has $\log \int_{M} e^{4 t v} d V_{g} \simeq 4 t \max _{M} v$. It follows that for $v \in \operatorname{ker} P_{g}$

$$
I I(t v)=4 t\left(\int_{M} Q v d V_{g}-k_{P} \max _{M} v\right)+o(t), \quad t \rightarrow+\infty .
$$

It would be useful to prove that this function either has constant sign on the unit sphere of ker $P_{g}$, or that its negative sublevel has non-trivial topology. Under these conditions one might hope to adapt the variational scheme in [24] and prove existence of solutions to (3). 
Also the case $k_{p}=8 k \pi^{2}$ should require some new geometric insight. In the recent paper [36] the authors considered four manifolds such that $k_{p}=8 \pi^{2}$. They approached the problem perturbing the equation as in (10), letting $\rho$ tending to 1 from below: indeed for $\rho<1$ by the Adams inequality $I I_{\rho}$ admits a minimizer $u_{\rho}$. In [36] the asymptotic behavior of $u_{\rho}$ was studied and it was shown that if the function

$$
\left(\Delta_{g, y} S(x, y)\right)_{y=x}+4\left|\nabla_{g, y} S(x, y)_{y=x}\right|^{2}-\frac{R_{g}(x)}{8}
$$

is positive on $M$, then $u_{\rho}$ converges to a solution of (3). In the above formula $S$ stands for the regular part of the Green's function of $P_{g}$ : precisely, using the convention that

$$
P_{g} G(x, y)+2 Q_{g}(y)=16 \pi^{2} \delta_{x}
$$

one has that, near the point $x, G$ satisfies

$$
G(x, y)=-2 \log \operatorname{dist}(x, y)+S(x, y)
$$

for some $C^{2}$-smooth $S$. However, there are so far no known conditions which can guarantee the positivity of the function in (11). A result of this kind would be a counterpart of the positive mass theorem by Schoen and Yau for compact manifolds of positive scalar curvature.

\section{Acknowledgements}

The author has been supported by M.U.R.S.T within the PRIN 2006 Variational Methods and Nonlinear Differential Equations.

\section{References}

[1] Adams D., A sharp inequality of J. Moser for higher order derivatives, Ann. of Math. (2) 128 (1988), 385-398.

[2] Bahri A., Critical points at infinity in some variational problems, Research Notes in Mathematics, Vol. 182, Longman-Pitman, London, 1989.

[3] Bahri A., Coron J.M., On a nonlinear elliptic equation involving the critical Sobolev exponent: the effect of the topology of the domain, Comm. Pure Appl. Math. 41 (1988), 253-294.

[4] Branson T.P., The functional determinant, Global Analysis Research Center Lecture Note Series, No 4, Seoul National University, 1993.

[5] Branson T.P., Differential operators canonically associated to a conformal structure, Math. Scand. 57 (1985), 293-345.

[6] Branson T.P., Gover A.R., Conformally invariant operators, differential forms, cohomology and a generalisation of Q-curvature, Comm. Partial Differential Equations 30 (2005), 1611-1669, math.DG/0309085.

[7] Branson T.P., Ørsted B., Explicit functional determinants in four dimensions, Proc. Amer. Math. Soc. 113 (1991), 669-682.

[8] Branson T.P., Chang S.Y.A., Yang P.C., Estimates and extremal problems for the log-determinant on 4-manifolds, Comm. Math. Phys. 149 (1992), 241-262.

[9] Bredon G.E., Topology and geometry, Graduate Texts in Mathematics, Vol. 139, Springer, 1997.

[10] Brendle S., Global existence and convergence for a higher order flow in conformal geometry, Ann. of Math. (2) 158 (2003), 323-343, math.DG/0404415.

[11] Brendle S., Prescribing a higher order conformal invariant on $S^{n}$, Comm. Anal. Geom. 11 (2003), 837-858.

[12] Chang S.Y.A., Gursky M.J., Yang P.C., An equation of Monge-Ampère type in conformal geometry, and four-manifolds of positive Ricci curvature, Ann. of Math. (2) 155 (2002), 709-787, math.DG/0409583.

[13] Chang S.Y.A., Gursky M.J., Yang P.C., A conformally invariant sphere theorem in four dimensions, Publ. Math. Inst. Hautes Études Sci. 98 (2003), 105-143, math.DG/0309287. 
[14] Chang S.Y.A., Qing J., The zeta functional determinants on manifolds with boundary. I. The formula, J. Funct. Anal. 147 (1997), 327-362.

[15] Chang S.Y.A., Qing J., The zeta functional determinants on manifolds with boundary. II. Extremal metrics and compactness of isospectral set, J. Funct. Anal. 147 (1997), 363-399.

[16] Chang S.Y.A., Qing J., Yang P.C., Compactification of a class of conformally flat 4-manifold, Invent. Math. 142 (2000), 65-93.

[17] Chang S.Y.A., Qing J., Yang P.C., On the Chern-Gauss-Bonnet integral for conformal metrics on $\mathbf{R}^{4}$, Duke Math. J. 103 (2000), 523-544.

[18] Chang S.Y.A., Yang P.C., Extremal metrics of zeta functional determinants on 4-manifolds, Ann. of Math. (2) 142 (1995), 171-212.

[19] Chang S.Y.A., Yang P.C., On a fourth order curvature invariant, in Spectral Problems in Geometry and Arithmetic, Editor T. Branson, Comtemp. Math. 237 (1999), 9-28.

[20] Chen C.C., Lin C.S., Topological degree for a mean field equation on Riemann surfaces, Comm. Pure Appl. Math. 56 (2003), 1667-1727.

[21] Chen W., Li C., Prescribing Gaussian curvatures on surfaces with conical singularities, J. Geom. Anal. 1 (1991), 359-372.

[22] Ding W., Jost J., Li J., Wang G., Existence results for mean field equations, Ann. Inst. H. Poincaré Anal. Non Linéaire 16 (1999), 653-666, dg-ga/9710023.

[23] Djadli Z., Malchiodi A., A fourth order uniformization theorem on some four manifolds with large total Q-curvature, C. R. Math. Acad. Sci. Paris 340 (2005), 341-346.

[24] Djadli Z., Malchiodi A., Existence of conformal metrics with constant Q-curvature, Ann. of Math. (2), to appear, math.DG/0410141.

[25] Druet O., Robert F., Bubbling phenomena for fourth-order four-dimensional pdes with exponential growth, Proc. Amer. Math. Soc. 134 (2006), 897-908.

[26] Fefferman C., Graham C.R., Q-curvature and Poincaré metrics, Math. Res. Lett. 9 (2002), $139-151$.

[27] Fefferman C., Hirachi K., Ambient metric construction of $Q$-curvature in conformal and CR geometries, Math. Res. Lett. 10 (2003), 819-832, math.DG/0303184.

[28] Gover A.R., Invariants and calculus for conformal geometry, Adv. Math. 163 (2001), 206-257.

[29] Gover A.R., Peterson L.J., The ambient obstruction tensor and the conformal deformation complex, Pacific J. Math. 226 (2006), 309-351, math.DG/0408229.

[30] Graham C.R., Jenne R., Mason L.J., Sparling G., Conformally invariant powers of the Laplacian. I. Existence, J. London Math. Soc. 46 (1992), 557-565.

[31] Graham C.R., Juhl A., Holographic formula for $Q$-curvature, arXiv:0704.1673.

[32] Graham C.R., Zworski M., Scattering matrix in conformal geometry, Invent. Math. 152 (2003), 89-118, math.DG/0109089.

[33] Gursky M., The Weyl functional, de Rham cohomology, and Kahler-Einstein metrics, Ann. of Math. (2) 148 (1998), 315-337.

[34] Gursky M., The principal eigenvalue of a conformally invariant differential operator, with an application to semilinear elliptic PDE, Comm. Math. Phys. 207 (1999), 131-143.

[35] Gursky M., Viaclovsky J., A fully nonlinear equation on four-manifolds with positive scalar curvature, J. Differential Geom. 63 (2003) 131-154, math.DG/0301350.

[36] Li J., Li Y., Liu P., The $Q$-curvature on a 4-dimensional Riemannian manifold $(M, g)$ with $\int_{M} Q d V_{g}=8 \pi^{2}$, math.DG/0608543.

[37] Malchiodi A., Compactness of solutions to some geometric fourth-order equations, J. Reine Angew. Math. 594 (2006), 137-174, math.AP/0410140.

[38] Malchiodi A., Morse theory and a scalar field equation on compact surfaces, Preprint.

[39] Malchiodi A., Struwe M., Q-curvature flow on $S^{4}$, J. Differential Geom. 73 (2006), 1-44.

[40] Ndiaye C.B., Constant Q-curvature metrics in arbitrary dimension, J. Funct. Anal., to appear.

[41] Ndiaye C.B., Conformal metrics with constant $Q$-curvature for manifolds with boundary, Preprint, 2007.

[42] Ndiaye C.B., Constant T-curvature conformal metrics on 4-manifolds with boundary, arXiv:0708.0732. 
[43] Paneitz S., A quartic conformally covariant differential operator for arbitrary pseudo-Riemannian manifolds, Preprint, 1983.

[44] Struwe M., The existence of surfaces of constant mean curvature with free boundaries, Acta Math. 160 (1988), 19-64.

[45] Struwe M., Variational methods. Applications to nonlinear partial differential equations and Hamiltonian systems, 3rd ed., Springer-Verlag, Berlin, 2000.

[46] Wei J., Xu X., On conformal deformations of metrics on $S^{n}$, J. Funct. Anal 157 (1998), 292-325. 